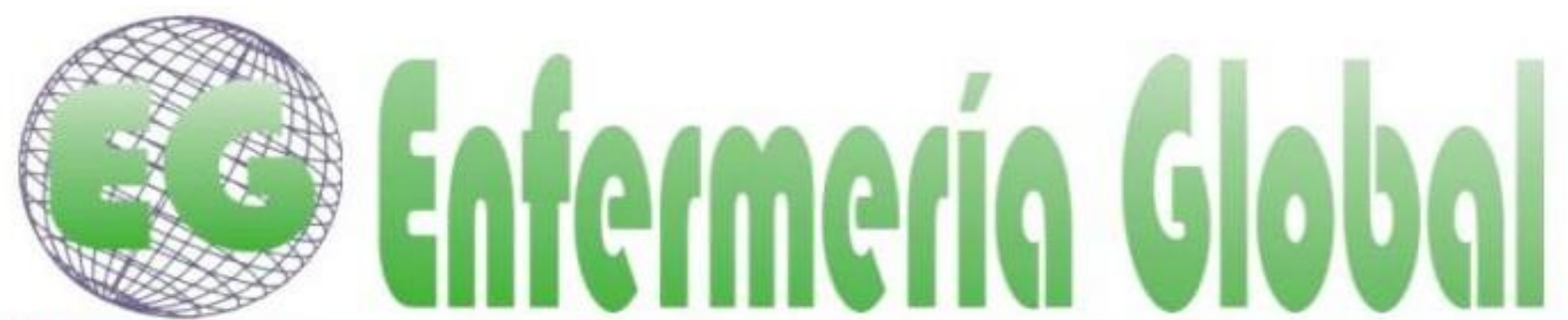

\title{
ORIGINALES
}

\section{Perspectiva espiritualidad en integrantes de alcohólicos anónimos: estudio piloto}

Perspective spirituality in members of alcoholics anonymous: pilot study

Linda Azucena Rodríguez-Puente ${ }^{1}$

María Magdalena Alonso-Castillo ${ }^{2}$

Javier Álvarez-Bermudes ${ }^{3}$

Marco Vinicio Gómez-Meza ${ }^{4}$

Nora Angélica Armendáriz-García ${ }^{1}$

Eva Kerena Hernández Martínez ${ }^{5}$

${ }^{1}$ Doctora en Ciencias de Enfermería. Facultad de Enfermería, Universidad Autónoma de Nuevo León. México.

${ }^{2}$ Doctora en Filosofía en Psicología. Facultad de Enfermería, Universidad Autónoma de Nuevo León. México.

${ }^{3}$ Doctor en Psicología Social. Facultad de Psicología, Universidad Autónoma de Nuevo León. México. 4Doctor en Estadística. Facultad de Economía, Universidad Autónoma de Nuevo León. México.

${ }^{5}$ Maestra en Ciencias de Enfermería. Profesor Titular Facultad de Enfermería "Dr. Santiago Valdés Sánchez", Universidad Autónoma de Coahuila. México.

E-mail:Irpuente88@hotmail.com

http://dx.doi.org/10.6018/eglobal.16.3260831

Recibido: $10 / 06 / 2016$

Aceptado: 30/08/2016

\section{RESUMEN:}

La espiritualidad es un concepto que se ha relacionado de manera positiva con la salud física y mental de las personas y se ha observado que juega un papel importante en el manejo y recuperación del abuso de sustancias como el alcohol.

El objetivo principal de este estudio fue determinar la perspectiva espiritual de los integrantes de AA (Alcohólicos anónimos).

El diseño fue cuantitativo, descriptivo de corte transversal. La población estuvo conformada por integrantes de AA de 3 municipios suburbanos de Nuevo León, México. Se realizó un muestreo no probabilístico, la muestra estuvo conformada por 35 adultos pertenecientes a los grupos de AA, se utilizó la Escala de Perspectiva Espiritual (SPS).

Los resultados muestran que el promedio del índice de espiritualidad en los participantes fue de 66.33 $(D E=17.23)$, el promedio de prácticas espirituales fue de $59.64(D E=25.11)$ y el de creencias espirituales fue de $70.70(D E=16.60)$.

Se concluye que los integrantes de AA tienen un nivel alto de espiritualidad total, en prácticas espirituales y en creencias espirituales.

Palabras clave: Espiritualidad; Alcohólicos Anónimos; Alcoholismo 


\section{ABSTRACT:}

Spirituality is a concept that has been linked positively with physical and mental health of people and has been found to play an important role in the management and recovery of substance abuse like alcohol. The main objective of this study was to determine the spiritual perspective of the members of AA. The design was quantitative, descriptive cross-sectional. The population consisted of members of AA 3 suburban municipalities of Nuevo Leon, Mexico. A non-probability sampling was performed, the sample consisted of 35 adults from AA groups, Spiritual Perspective Scale (SPS) was used. The results show that the average rate of spirituality in the participants was $66.33(S D=17.23)$, the average spiritual practices was $59.64(S D=25.11)$ and spiritual belief was $70.70(S D=16.60)$. It is concluded that AA members have a high level of total spirituality, spiritual practices and spiritual beliefs.

Keywords: Spirituality; Alcoholics Anonymous; Alcoholism

\section{INTRODUCCIÓN}

La espiritualidad es un concepto que se ha relacionado de manera positiva con la salud física y mental de las personas y se ha observado que juega un papel importante en el manejo y recuperación del abuso de sustancias como el alcohol ${ }^{1,2}$.

En el caso de la dependencia al alcohol y el alcoholismo la espiritualidad representa un concepto de gran importancia. Diferentes investigaciones señalan que la espiritualidad puede actuar como factor protector de la dependencia al alcohol, favorece en el tratamiento de la recuperación, se relaciona con periodos largos de abstinencia y ayuda en el manejo del craving ${ }^{2,3,4,5}$.

En México, la Encuesta Nacional de Adicciones (2011) reporta que el 6\% de la población desarrolló dependencia al alcohol, lo que equivale a 4.9 millones de personas de 12 a 65 años de edad, de los cuales el $10.8 \%$ corresponde a hombres y el $1.8 \%$ a mujeres. La dependencia al alcohol afecta al $4.1 \%$ de los adolescentes y al $6.6 \%$ de los adultos, esta proporción aumentó significativamente entre los hombres del año 2008 a 2011, mostrando menor distancia entre hombres y mujeres adolescentes ( 3 hombres por cada mujer) que lo que se observa en adultos ( 7 hombres por cada mujer). La proporción de mujeres adultas con dependencia al alcohol es de $1.7 \%$ similar a la observada en mujeres adolescentes (2\%); en los hombres se presenta por cada dos adultos con dependencia al alcohol un adolescente con esta condición ${ }^{6}$.

Actualmente, AA (Alcohólicos Anónimos) representa el recurso terapéutico más difundido para tratar el problema del alcoholismo en México y otros países sin afirmar que sea el más efectivo. La adherencia o afiliación en AA puede ser benéfica para personas que viven esta problemática y se destaca como constructo que apoya el proceso de recuperación de AA la asistencia a reuniones y el nivel de participación en los grupos AA y se indica que cuando mayor es el involucramiento con el programa de doce pasos mejor es el pronóstico de recuperación $\mathrm{n}^{4,7,8,9}$.

Así mismo, Alcohólicos Anónimos (AA) y su programa de 12 pasos atribuye una gran importancia al concepto de la espiritualidad. AA en su programa supone que el alcoholismo es una enfermedad del espíritu, es por ello que los 12 pasos rectores de AA se encuentran vinculados a la espiritualidad y acentúan principios como la oración, la meditación y la aceptación de un "Dios" definido de manera personal o "Poder Superior ${ }^{2,10,11 .}$

La espiritualidad ha sido definida por Reed como la perspectiva personal y los comportamientos que expresan sentido de pertenencia (relación) a una dimensión 
trascendente o a algo más grande que sí mismo. Desde ésta perspectiva espiritual, conceptos como "orar" y "Ser Supremo" son significativos para la construcción de este constructo. Específicamente dentro de los indicadores de la espiritualidad podemos encontrar la oración, significado a la vida, lectura y contemplación, sentido de cercanía a un Ser Supremo, interacción con otros y otras experiencias que puedan reflejar interacción o participación espiritual ${ }^{1}$.

Estudios realizados en AA señalan que la espiritualidad se relaciona de manera positiva con el porcentaje de días de abstinencia, predice el no consumo de alcohol y que el "despertar espiritual eleva 2 veces la probabilidad de mantener la abstinencia en los integrantes de $A^{2,3,4}$.

Sin embargo, en México son pocos los estudios realizados para conocer el nivel de perspectiva espiritual en esta población. Para el personal de enfermería es de suma importancia conocer aquellos factores que favorecen la abstinencia en los integrantes de AA para el desarrollo de intervenciones basadas en la evidencia científica enfocadas en el reforzamiento de recursos y habilidades que favorezcan el no consumo de alcohol en personas dependientes de alcohol que se encuentran en tratamiento. Cabe señalar que en nuestro país no se han encontrado estudios realizados por profesionales de enfermería donde se aborde esta variable en población de AA. Así mismo, no se han localizado estudios donde se utilice la Escala de Perspectiva Espiritual (SPS) ${ }^{1}$ en esta población.

Por lo tanto, el objetivo principal de este trabajo es determinar la perspectiva espiritual en integrantes de AA. Un estudio piloto.

\section{Objetivos específicos}

1. Determinar el tiempo de llenado de la Escala de Perspectiva Espiritual (SPS) en integrantes de AA.

2. Determinar el Coeficiente de Confiabilidad de Alpha de Cronbach de la Escala de Perspectiva Espiritual (SPS) en integrantes de AA.

3. Describir las frecuencias y proporciones de prácticas y creencias espirituales de acuerdo a la Escala de Perspectiva Espiritual (SPS) en integrantes de AA.

\section{MATERIAL Y MÉTODOS}

\section{Diseño de estudio}

El diseño fue cuantitativo-descriptivo. La población estuvo conformada por integrantes de AA de 3 municipios suburbanos de Nuevo León, México. Se realizó un muestreo no probabilístico, la muestra estuvo conformada por 35 adultos pertenecientes a los grupos de AA.

\section{Mediciones}

Datos sociodemográficos. Cédula de Datos Personales (CDP) que consta de 8 preguntas donde se indaga el sexo, edad, estado civil, nivel educativo, ocupación, la religión y la edad de ingreso al programa de AA y la cantidad de recaídas.

Espiritualidad. La Escala de Perspectiva Espiritual (SPS) ${ }^{1}$, mide un conocimiento de sí mismo, a un sentido de conexión con un ser de naturaleza superior o a la existencia de un propósito supremo. El instrumento se basa en la conceptualización de 
espiritualidad como una experiencia humana particularmente relevante en las fases tardías del desarrollo de la vida y en momentos donde se aumenta la conciencia de la mortalidad.

La escala consta de 10 enunciados que se califican en un rango de 1 a 6 para un puntaje total de 60. Las cuatro primeras preguntas corresponden a la subescala de prácticas espirituales y se responden con los siguientes criterios: 1) Nunca, 2) Menos de una vez al año, 3) Más o menos una vez al año, 4) Más o menos una vez al mes, 5) Más o menos una vez a la semana y 6) Más o menos una vez al día. Las preguntas del 5 al 10 corresponden a la subescala de creencias espirituales y son respondidas de la siguiente manera: 1) Extremadamente en desacuerdo, 2) Desacuerdo, 3) En desacuerdo más que en acuerdo, 4) De acuerdo más que en desacuerdo, 5) De acuerdo y 6) Extremadamente de acuerdo.

Se obtuvieron índices de cada subescala y de la escala en general, donde a mayor puntaje mayor es el índice de espiritualidad. Para clasificar el nivel de perspectiva espiritual de los participantes se tomaron los niveles establecidos por Vázquez ${ }^{12}$, el cual considera los siguientes: bajo nivel de espiritualidad de 10-30 puntos, nivel moderado de 31-45 puntos, y nivel alto de espiritualidad de 46-60 puntos.

La escala original reporta Alpha de Cronbach de .90, un estudio realizado en población latina ${ }^{13}$, la escala reportó un Alpha de Cronbach de .89.

\section{Procedimiento de recolección de información}

Para iniciar con la implementación de este estudio se obtuvo la aprobación de las Comisiones de Ética para la Investigación y la de Investigación de la Facultad de Enfermería de la Universidad Autónoma de Nuevo León (FAEN-D-1182).

Se acudió a 3 centros AA para solicitar la autorización de cada grupo y de sus integrantes, se les explicó el objetivo del estudio y a aquellos que aceptaron participar se les entregó el consentimiento informado. Posteriormente, se les entregó un sobre cerrado con los instrumentos y procedieron al llenado de los cuestionarios reforzando el carácter confidencial y anónimo de la información. Por último, se les pidió, que al término del llenado, depositaran el sobre cerrado sin identificación en una caja sellada que se encontraba en la parte delantera del espacio físico donde se reúne cada grupo. Al depositar el sobre con los instrumentos, se les agradeció el tiempo y la información proporcionada.

\section{Análisis de datos}

Se realizó a través del paquete estadístico SPSS para ciencias sociales versión 20. Se utilizó estadística descriptiva. El análisis descriptivo de las variables continuas y categóricas se realizó mediante frecuencias, proporciones y medidas de tendencia central y de variabilidad. Además, se determinó la Confiabilidad del instrumento a través del Alpha de Cronbach.

\section{RESULTADOS}

En relación a los datos sociodemográficos la media de edad de los participantes fue de $51.03(D E=13.82)$ años de edad, la mayoría era de sexo masculino en un $97.1 \%$ (34), el $37.1 \%$ eran solteros, el $31.4 \%$ habían cursado hasta nivel primaria, la mayoría trabaja (62.9\%) y predominó la religión católica en un $68.6 \%$. Respecto a la edad de 
ingreso al $A A$, el promedio fue de 35.06 años $(D E=9.81)$ y promedio de recaídas fue de $4.17(D E=10.08)$.

El tiempo de llenado del instrumento fue de 30 minutos incluyendo la CDP. El Alpha de Cronbach de la Escala de Perspectiva Espiritual (SPS) para este estudio fue de .90 .

El 37.1\% (13) de los participantes refirieron mencionar asuntos espirituales cuando están hablando con su familia o amigos más o menos una vez al mes, el $37.1 \%$ (13) mencionó que más o menos una vez al día comparte usted con otros los problemas y alegrías de vivir de acuerdo con sus creencias espirituales, el $40.0 \%$ (14) lee materiales espirituales más o menos una vez al día y el $60.0 \%$ reza u ora en privado 0 hace meditación más o menos una vez al día (Tabla I).

Tabla I.-Frecuencias y proporciones de las prácticas espirituales

\begin{tabular}{|c|c|c|c|c|c|c|c|c|c|c|c|c|}
\hline & \multicolumn{2}{|c|}{1} & \multicolumn{2}{|c|}{2} & \multicolumn{2}{|c|}{3} & \multicolumn{2}{|c|}{4} & \multicolumn{2}{|c|}{5} & \multicolumn{2}{|c|}{6} \\
\hline & $f$ & $\%$ & $f$ & $\%$ & $f$ & $\%$ & $f$ & $\%$ & $f$ & $\%$ & $f$ & $\%$ \\
\hline $\begin{array}{l}\text { 1. Cuando está hablando } \\
\text { con su familia o sus } \\
\text { amigos, ¿cuántas veces } \\
\text { menciona usted asuntos } \\
\text { espirituales? }\end{array}$ & 6 & 17.1 & 4 & 11.4 & 1 & 2.9 & 13 & 37.1 & 9 & 25.7 & 1 & 2.9 \\
\hline $\begin{array}{l}\text { 2. ¿Cuántas veces } \\
\text { comparte usted con otros } \\
\text { los problemas y alegrías } \\
\text { de vivir de acuerdo con } \\
\text { sus creencias } \\
\text { espirituales? }\end{array}$ & 5 & 14.3 & 2 & 5.7 & 1 & 2.9 & 2 & 5.7 & 12 & 34.3 & 13 & 37.1 \\
\hline $\begin{array}{l}\text { 3. ¿Cuántas veces lee } \\
\text { usted materiales de algo } \\
\text { espiritual? }\end{array}$ & 7 & 20.0 & 0 & 0.0 & 1 & 2.9 & 0 & 0.0 & 13 & 37.1 & 14 & 40.0 \\
\hline $\begin{array}{l}\text { 4. ¿Cuántas veces reza u } \\
\text { ora usted en privado o } \\
\text { hace meditación? }\end{array}$ & 3 & 8.6 & 1 & 2.9 & 2 & 5.7 & 0 & 0.0 & 8 & 22.9 & 21 & 60.0 \\
\hline
\end{tabular}

El 51.4\% (18) de los participantes está de acuerdo en que el perdón es una parte importante de su espiritualidad, el 45.7\% (16) está de acuerdo en ver la espiritualidad como una guía para tomar decisiones en su vida diaria, el $51.4 \%$ (18) está extremadamente de acuerdo en que sus creencias espirituales son una parte importante de su vida, el $57.1 \%$ está extremadamente de acuerdo en que frecuentemente se siente muy cerca de Dios, o a un "poder más grande" en momentos importantes de su vida diaria, el 54.3\% (19) está extremadamente de acuerdo en que sus creencias espirituales han influenciado su vida y el $51.4 \%$ (18) está extremadamente de acuerdo en que sus creencias espirituales son especialmente importantes porque responden a muchas de las preguntas que tiene sobre el significado de la vida (tabla II). 
Tabla II.-Frecuencias y proporciones de las creencias espirituales

\begin{tabular}{|c|c|c|c|c|c|c|c|c|c|c|c|c|}
\hline & \multicolumn{2}{|c|}{1} & \multicolumn{2}{|c|}{2} & \multicolumn{2}{|c|}{3} & \multicolumn{2}{|c|}{4} & \multicolumn{2}{|c|}{5} & \multicolumn{2}{|c|}{6} \\
\hline & $f$ & $\%$ & $f$ & $\%$ & $f$ & $\%$ & $f$ & $\%$ & $f$ & $\%$ & $f$ & $\%$ \\
\hline $\begin{array}{l}\text { 5. El perdón es una parte } \\
\text { importante de su } \\
\text { espiritualidad. }\end{array}$ & 0 & 0.0 & 1 & 2.9 & 0 & 0.0 & 2 & 5.7 & 18 & 51.4 & 14 & 40.0 \\
\hline $\begin{array}{l}\text { 6. Ve la espiritualidad } \\
\text { como una guía para tomar } \\
\text { decisiones en su vida } \\
\text { diaria. }\end{array}$ & 1 & 2.9 & 0 & 0.0 & 0 & 0.0 & 4 & 11.4 & 16 & 45.7 & 14 & 40.0 \\
\hline $\begin{array}{l}\text { 7. Sus creencias } \\
\text { espirituales son una parte } \\
\text { importante de su vida. }\end{array}$ & 2 & 5.7 & 0 & 0.0 & 0 & 0.0 & 1 & 2.9 & 14 & 40.0 & 18 & 51.4 \\
\hline $\begin{array}{l}\text { 8. Frecuentemente se } \\
\text { siente muy cerca de Dios, } \\
\text { o a un "poder mas } \\
\text { grande" en momentos } \\
\text { importantes de su vida } \\
\text { diaria }\end{array}$ & 2 & 5.7 & 0 & 0.0 & 0 & 0.0 & 2 & 5.7 & 11 & 31.4 & 20 & 57.1 \\
\hline $\begin{array}{l}\text { 9. Sus creencias } \\
\text { espirituales han } \\
\text { influenciado su vida }\end{array}$ & 2 & 5.7 & 0 & 0.0 & 0 & 0.0 & 1 & 2.9 & 13 & 37.1 & 19 & 54.3 \\
\hline $\begin{array}{l}\text { 10. Sus creencias } \\
\text { espirituales son } \\
\text { especialmente } \\
\text { importantes porque } \\
\text { responden a muchas de } \\
\text { las preguntas que tiene } \\
\text { sobre el significado de la } \\
\text { vida }\end{array}$ & 2 & 5.7 & 0 & 0.0 & 0 & 0.0 & 1 & 2.9 & 14 & 40.0 & 18 & 51.4 \\
\hline
\end{tabular}

El índice de espiritualidad en los participantes presentó un promedio de 66.33 ( $D E=$ 17.23), el promedio de prácticas espirituales fue de $59.64(D E=25.11)$ y el de creencias espirituales fue de $70.70(D E=16.60)$ los cuales son considerados como un nivel alto de espiritualidad(12) (tabla III).

Tabla III.-Índice de espiritualidad

\begin{tabular}{lcccc}
\hline & $n$ & $\bar{X}$ & $M d n$ & $D E$ \\
\hline Índice total de espiritualidad & 35 & 66.33 & 70.00 & 17.23 \\
Índice de prácticas espirituales & 35 & 59.64 & 66.67 & 25.11 \\
Índice de creencias espirituales & 35 & 70.79 & 72.22 & 16.60 \\
\hline
\end{tabular}

Nota: $n=$ muestra, $\bar{X}=$ media, $M d n=$ mediana $D E=$ desviación estándar

\section{DISCUSIÓN}

El objetivo principal de este estudio fue determinar la perspectiva espiritual en integrantes de $A A$, se encontró que los integrantes de AA tienen una perspectiva espiritual alta. Esto coincide con lo reportado por Kelly ${ }^{2}$ que menciona que las personas que asisten a AA tienen un nivel elevado de espiritualidad. Es posible que nivel espiritual en los participantes presente un nivel debido a que el programa de 12 pasos de AA se basa principalmente en la concepción de un "Ser Supremo" y promueve la espiritualidad en 11 de sus pasos. 
El programa conduce a la persona dependiente del alcohol (alcohólico) a que admita su impotencia frente al alcohol a través de la ayuda de un poder superior concebido de manera individual y particular (1 a 3 Pasos). Posteriormente se promueve realizar un examen de conciencia y un inventario moral, con la intención de reparar daños ocasionados a terceros durante la etapa alcohólica (4 a 9 Pasos). Después se hace énfasis a la continuidad de la auto-observación, la corrección permanente de errores y la dimensión espiritual (10 a 11 Pasos). Finalmente (paso 12) se promueve ayudar a otros alcohólicos o dependientes del alcohol a logar la sobriedad ${ }^{14}$.

Debido a que este es un estudio piloto, uno de los objetivos fue determinar el tiempo de llenado del SPS en integrantes de AA, se observó que los participantes tardaron aproximadamente en contestar el instrumento junto con la CDP, esto es similar a lo reportado por Galvin y Pérez ${ }^{15}$ en su estudio realizado en mujeres con cáncer. Es importante señalar que a la fecha no se han localizado estudios donde se utilice la SPS en población de AA, debido a esto fue necesario conocer la Confiabilidad del SPS en integrantes de AA.

La SPS mostró un Alpha de Cronbach de .90 el cual se considera aceptable y coincide con lo reportado por la autora de esta escala ${ }^{1}$ y superior con lo reportado en otras poblaciones ${ }^{15}$.

\section{CONCLUSIONES}

El tiempo de llenado del instrumento fue de treinta minutos.

La Escala de Perspectiva Espiritual (SPS) reportó para este estudio un Alpha de Cronbach de .90 .

Se reportaron niveles altos de perspectiva espiritualidad en la escala total, así como en la subescala de prácticas y creencias espirituales.

\section{Recomendaciones}

Se recomienda realizar una validación de la SPS en población de AA, así también seguir utilizando este instrumento en integrantes de AA para futuras investigaciones y realizar estudios donde se relacione esta variable con el consumo de alcohol, el tiempo de abstinencia y la cantidad de recaídas en integrantes de AA.

\section{REFERENCIAS}

1. Reed PG. Spirituality and well-being in terminally ill hospitalized adults. Research in $\begin{array}{llll}\text { Nursing } \quad \text { Health } & \text { 1987; } & \text { 335-344. }\end{array}$ http://www.ncbi.nlm.nih.gov/pubmed/3671781

2. Kelly JF, Stout RL, Magill M, Tonigan JS, Pagona ME. Spirituality in Recovery: A Lagged Mediational Analysis of Alcoholics Anonymous Principal Theoretical Mechanism of Behavior Change. Alcoholism Clinical and Experimental Research 2011, 35(3),

454-463.

http://www.ncbi.nlm.nih.gov/pmc/articles/PMC3117904/pdf/nihms246551.pdf

3. Robinson, E.A., Krentzman, A.R., Webb, J.R. \& Brower, K.J. Six-month changes in spirituality and religiousness in alcoholics predict drinking outcomes an nine months. Journal of Studies on Alcohol and Drugs 2011, 72(4), 660-668. https://www.researchgate.net/publication/51228543_Six- 
Month_Changes_in_Spirituality_and_Religiousness_in_Alcoholics_Predict_Drinking_ Outcomes_at_Nine_Months

4. Gutiérrez RR, Andrade PP, Jiménez TA, Saldivar HG, Juárez GF. Alcohólicos Anónimos: Aspectos relacionados con la adherencia (afiliación) y diferencias entre recaídas y no recaídas. Salud Mental 2009; 32 (5): 427-433. http://www.scielo.org.mx/pdf/sm/v32n5/v32n5a9.pdf

5. Mason SJ, Dean FP, Kelly PJ, Crowe TP. Do Spirituality and Religiosity Help

in the Management of Cravings in Substance Abuse Treatment? Substance Use \& Misuse 2009;

http://ro.uow.edu.au/cgi/viewcontent.cgi?article=1996\&context=hbspapers

6. Medina M. Instituto Nacional de Psiquiatría Ramón de la Fuente Muñiz; Instituto Nacional de Salud Pública; Secretaría de Salud. Encuesta Nacional de Adicciones 2011, Alcohol. Centro Nacional para la Prevención y el Control de Adicciones México, D.F. 2011.

7. Greenfield BL, Tonigan JS. The general alcoholics anonymous tools of recovery: The adoption of 12-Step practices and beliefs. Psychol Addict Behav 2013; 27 (3): 553-561. http://www.ncbi.nlm.nih.gov/pmc/articles/PMC3707937/

8. Rosovsky H. Alcohólicos Anónimos. En: Beber de tierra generosa. Ciencias de las bebidas alcohólicas en México. Fundación de Investigaciones Sociales AC (eds); México: 1998.

9. Rosovsky H, Leyva G. Movimiento de alcohólicos anónimos en México. Anales 1990; (1): 5-8.

10. Alcoholics Anonymous. Twelve steps and twelve traditions. Alcoholics Anonymous World Services; New York: 1952.

11. Alcoholics Anonymous. Alcoholics Anonymous: The story of how thousands of men and women have recovered from alcoholism. 4. Alcoholics Anonymous World Services; New York: 2001.

12. Vázquez $\mathrm{O}$, Whetsell $M$, Zavala MR. Bienestar espiritual y ansiedad en pacientes $\begin{array}{lllll}\text { diabéticos. } & \text { Aquichán } & 2006 ; & 6 & \text { (1): }\end{array}$ http://www.redalyc.org/articulo.oa?id=74160103

13. Campesino M, Belyea M, Schwartz G. Spirituality and cultural identification among latino and non-latino college students. Hispanic Health Care International 2009; 7 (2): 1-13. http://www.ncbi.nlm.nih.gov/pmc/articles/PMC2822391/

14. Alcoholics Anonymous. 2011 Membership Survey. Alcoholics Anonymous World Services; New York: 2012.

15. Galvis LMA, Pérez GB. Perspectiva espiritual de la mujer con cáncer. Aquichán 2011; $11 \quad$ (3): 256-273. http://aquichan.unisabana.edu.co/index.php/aquichan/article/view/2016/pdf

ISSN 1695-6141

(C) COPYRIGHT Servicio de Publicaciones - Universidad de Murcia 